\title{
Editorial
}

\section{Promoting research in periphery and improving transparency in scientific evaluation process.}

\author{
SunethAgampodisunethagampodi@yahoo.com \\ Sisira Siribaddanasisira.siribaddana@gmail.com
}

DOI: http://dx.doi.org/10.4038/amj.v9i2Supp.7593

This year, we had the largest number of submissions for free papers in the history of the Annual Academic Sessions (AAS) of the Anuradhapura Clinical Society (ACS). A total of 46 submissions were received through the online submission system. Not only the number of submissions, the quality and scope of the submitted papers were impressive. We had case reports as well as randomized control trials, and researchers representing regions from Jaffna to Galle.

We did a trial of a fully transparent online submission system this year, thanks to the free online conference management system available at http://www.easychair.org Through this system, we had a fully blinded peer review system which does not allow any changes to be made to the comments or marks provided by reviewers. If conference organizers declare a conflict of interest, papers authored by them are not visible and they are unable to influence or make changes to them. The President of the ACS had full access and has the full authority to control online activities by all others in the scientific committee. All abstracts submitted to AAS of the ACS 2015 are given a DOI (digital object identifier) and indexed in Google Scholar, DOAJ and have open access.

At least two reviewers reviewed all papers. We invited three reviewers for each paper, but only 12 papers received reviews from all three. The reviewers always included one reviewer from the hospital and one from the University. The review process was structured and the final decision was made based on the average of the scores given by the reviewers. All reviewers' comments were made available for authors to improve their submission.

On behalf of the scientific committee we would like to thank the free paper committee members, Buddhika Wijerathne and Jagath Ranasinghe, for their effort to make this year's AAS a success. We would also like to offer our gratitude to the members of the scientific committee listed below.

\section{Scientific Committee}

Daminda Dumingoarachchi: Consultant ENT Surgeon, Teaching Hospital Anuradhapura

Sanjaya Fernando: Consultant Pediatrics Neurologist, Teaching Hospital Anuradhapura

Asantha Jayawardane: Senior Lecturer, Department of Anatomy, Faculty of Medicine and Allied Sciences,Rajarata University of Sri Lanka

Sudheera Kalupahana: Professor in Human Nutrition, Department of Physiology Faculty of Medicine, University of Peradeniya.

UdithamalaRathnayake: Consultant Radiologist, Teaching Hospital Anuradhapura

VY Sabaratnam: Consultant Surgeon, Teaching Hospital Anuradhapura

Subhashinie Senadheera: Senior Lecturer, Department of Biochemistry, Faculty of Medicine and Allied Sciences, Rajarata University of Sri Lanka

Udaya De Silva: Consultant Neonatologist, Teaching Hospital Anuradhapura

Harshi Weerakoon: Senior Lecturer, Department of Biochemistry, Faculty of Medicine and Allied Sciences, Rajarata University of Sri Lanka

Kosala Weerakoon: Senior Lecturer, Department of Parasitology, Faculty of Medicine and Allied Sciences, Rajarata University of Sri Lanka

Sujanthi Wickramage: Senior Lecturer, Department of Physiology, Faculty of Medicine and Allied Sciences, Rajarata University of Sri Lanka

Nuwan Darshana Wickramasinghe: Senior Lecturer, Department of Community Medicine, Faculty of Medicine and Allied Sciences, Rajarata University of Sri Lanka 